\title{
SEPARABLE TERM STRUCTURES AND THE MAXIMAL DEGREE PROBLEM
}

\author{
DAMIR FILIPOVIĆ
}

\begin{abstract}
This paper discusses separable term structure diffusion models in an arbitrage-free environment. Using general consistency results we exploit the interplay between the diffusion coefficients and the functions determining the forward curve. We introduce the particular class of polynomial term structure models. We formulate the appropriate conditions under which the diffusion for a quadratic term structure model is necessarily an Ornstein-Uhlenbeck type process. Finally, we explore the maximal degree problem and show that basically any consistent polynomial term structure model is of degree two or less.
\end{abstract}

\section{INTRODUCTION}

This paper discusses arbitrage-free separable term structure (STS) models

$$
r(t, x)=\sum_{i=1}^{n} g_{i}(x) \phi_{i}\left(Z_{t}\right) .
$$

Here $\left\{r(t, x) \mid x \in \mathbb{R}_{+}\right\}$denotes the time $t$ forward rate curve, $Z$ is an $m$-dimensional diffusion process, and $g=\left(g_{1}, \ldots, g_{n}\right)$ and $\phi=\left(\phi_{1}, \ldots, \phi_{n}\right)$ are deterministic functions, for some $m, n \in \mathbb{N}$. Using general consistency results from Filipović (2001a), we exploit the interplay between the diffusion coefficients and the functions $g$ and $\phi$. As a byproduct we derive that the forward curve $x \mapsto r(t, x)$ is necessarily analytic.

Then we focus on the subclass of polynomial term structure (PTS) models

$$
r(t, x)=\sum_{|\mathbf{i}|=0}^{n} g_{\mathbf{i}}(x)\left(Z_{t}\right)^{\mathbf{i}},
$$

where we use the multi-index notation $\mathbf{i}=\left(i_{1}, \ldots, i_{m}\right),|\mathbf{i}|=i_{1}+\cdots+i_{m}$ and $z^{\mathbf{i}}=z_{1}^{i_{1}} \cdots z_{m}^{i_{m}}$. Here $n$ denotes the degree of the PTS; that is, there exists an index $\mathbf{i}$ with $|\mathbf{i}|=n$ and $g_{\mathbf{i}} \neq 0$.

For $n=1$ the PTS model (2) is actually an affine term structure (ATS) model. ATS models have been intensively studied by many authors. We refer to Duffie et al. $(1996,2000,2001)$ (see also references therein) and Section 3.1 in Filipović (2001a) . It is well known by now that, under the appropriate technical assumptions, $Z$ is necessarily a square-root process.

The next interesting case is $n=2$ : quadratic term structure (QTS) models. There has recently been both theoretical and empirical interest in QTS models, see Boyle and Tian (1999), Leippold and Wu (2002) and Gombani and Runggaldier

Date: August 24, 2000 (first draft); April 10, 2002 (this draft).

Key words and phrases. affine and quadratic term structures, interest rate models, forward curves, maximal degree of a polynomial term structure. 
(2000). We formulate the appropriate conditions under which $Z$ is necessarily an Ornstein-Uhlenbeck type process.

Finally, we explore a problem regarding the maximal degree $n$ of a PTS model. We show that, under mild technical assumptions, $n$ has to be less than or equal 2 . ATS and QTS models are, roughly speaking, the only consistent PTS models.

The outline of the paper is as follows. In Section 2 we provide the stochastic setup for $Z$ and prove general consistency results for STS models. Section 3 specializes these results for PTS models and exploits the maximal degree problem. We conclude by Section 4 .

\section{Separable Term Structures}

Let $\left(\Omega, \mathcal{F},\left(\mathcal{F}_{t}\right)_{t \in \mathbb{R}_{+}}, \mathbb{Q}\right)$ denote a filtered complete probability space, satisfying the usual conditions, and let $W$ be a standard $d$-dimensional $\left(\mathcal{F}_{t}\right)$-Brownian motion, $d \in \mathbb{N}$. Fix a closed set $\mathcal{Z} \subseteq \mathbb{R}^{m}$ with non-empty interior, $m \in \mathbb{N}$, and denote by $b$ and $\sigma$ two continuous mappings from $\mathcal{Z}$ into $\mathbb{R}^{m}$ and $\mathbb{R}^{m \times d}$, respectively. We assume that for every $z_{0} \in \mathcal{Z}$ there exists a unique continuous $\mathcal{Z}$-valued strong solution $Z=Z^{z_{0}}$ of

$$
\begin{aligned}
d Z_{t} & =b\left(Z_{t}\right) d t+\sigma\left(Z_{t}\right) d W_{t} \\
Z_{0} & =z_{0} .
\end{aligned}
$$

Remark 2.1. Notice that $b$ and $\sigma$ may be time varying, by setting $Z_{t}^{1}=t$.

We shall first discuss the general STS model (1), where $g \in C^{0}\left(\mathbb{R}_{+} ; \mathbb{R}^{n}\right)$ and $\phi \in C^{2}\left(\mathcal{Z} ; \mathbb{R}^{n}\right)$, for some $n \in \mathbb{N}$. We say that $\mathbb{Q}$ is a local martingale measure for $z_{0} \in \mathcal{Z}$ if all discounted bond price processes

$$
\left(e^{-\int_{0}^{t} r(s, 0) d s} e^{-\int_{0}^{T-t} r(t, x) d x}\right)_{t \in[0, T]}, \quad T \in \mathbb{R}_{+},
$$

are $\mathbb{Q}$-local martingales for $Z=Z^{z_{0}}$. We introduce the consistency condition

(C): $\mathbb{Q}$ is a local martingale measure for every $z_{0} \in \mathcal{Z}$.

It is standard to consider (C) as a necessary condition for the absence of arbitrage. Under (C) there is a functional interplay between $g, \phi, b$ and $\sigma$. Write

$$
G(x):=\int_{0}^{x} g(y) d y
$$

and define

$$
\begin{aligned}
\Gamma_{k}(x, z) & :=\sum_{i=1}^{n} G_{i}(x) \frac{\partial \phi_{i}(z)}{\partial z_{k}} \\
\Lambda_{k l}(x, z)=\Lambda_{l k}(x, z) & :=\sum_{i=1}^{n} G_{i}(x) \frac{\partial^{2} \phi_{i}(z)}{\partial z_{k} \partial z_{l}} .
\end{aligned}
$$

The following theorem is a restatement of the general consistency results in Filipovic (2001a). It is a particular form of the Heath-Jarrow-Morton drift condition (Heath et al. (1992)). We denote by $a:=\sigma \sigma^{*}$ the diffusion function of $Z$. 
Theorem 2.2. Under the above assumptions, (C) holds if and only if

$$
\begin{aligned}
\sum_{i=1}^{n}\left(g_{i}(x)-g_{i}(0)\right) \phi_{i}(z)= & \sum_{k=1}^{m} b_{k}(z) \Gamma_{k}(x, z) \\
& +\frac{1}{2} \sum_{k, l=1}^{m} a_{k l}(z)\left(\Lambda_{k l}(x, z)-\Gamma_{k}(x, z) \Gamma_{l}(x, z)\right),
\end{aligned}
$$

for all $(x, z) \in \mathbb{R}_{+} \times \mathcal{Z}$.

From now on we assume that $(\mathbf{C})$ is satisfied.

Of course, any STS model (1) can be considered as an ATS model with $n$ factors $y_{i}=\phi_{i}(z)$ (albeit the state space $\phi(\mathcal{Z})$ can have empty interior). But an ATS model cannot in general be realized in the form (1) for a given $\phi$. This can be seen from the following example, where $m=1, n=2$ and $\phi(z)=\left(z, z^{2}\right)$. Set $g_{1}(x) \equiv 1$ and $g_{2}(x)=x$. It is shown in Filipović (2001b) that there exists a consistent ATS model

$$
r(t, x)=Y_{t}^{1}+x Y_{t}^{2},
$$

where $Y=\left(Y^{1}, Y^{2}\right)$ is a non-degenerate diffusion. It is impossible, however, to have $Y_{t}^{1}=Z_{t}$ and $Y_{t}^{2}=\left(Z_{t}\right)^{2}$ for a real-valued diffusion $Z$. Indeed, (6) yields for $\phi_{1}(z)=z$ and $\phi_{2}(z)=z^{2}$

$$
x z^{2}=b(z)\left(x+x^{2} z\right)-\frac{1}{2} a(z)\left(2 x^{3} z+x^{4} z^{2}\right),
$$

which implies $b=a=0$.

The preceding example shows that $b$ and $a$ may be completely determined by $g$ and $\phi$. Pretending that $g$ and $\phi$ are known, we now want to invert equation (6) for $b$ and $a$. Moreover, we want to infer from (6) as much structural information on $b$ and $a$ in terms of $g$ and $\phi$ as possible, and vice versa.

Proposition 2.3. Suppose that the functions

$$
\Gamma_{k}(\cdot, z) \quad \text { and } \quad \Lambda_{k l}(\cdot, z)-\Gamma_{k}(\cdot, z) \Gamma_{l}(\cdot, z), \quad 1 \leq k \leq l \leq m,
$$

are linearly independent, for all $z \in \mathcal{D}$, where $\mathcal{D}$ is a dense subset of $\mathcal{Z}$. Then $b$ and $a$ are uniquely determined by $g$ and $\phi$.

Proof. Set $M=m+m(m+1) / 2$, the number of unknown functions $b_{k}$ and $a_{k l}=a_{l k}$. Let $z \in \mathcal{D}$. Then there exists a sequence $0 \leq x_{1}<\cdots<x_{M}$ such that the $M \times M$ matrix with $i$-th row vector built by $\Gamma_{k}\left(x_{i}, z\right)$ and $\Lambda_{k l}\left(x_{i}, z\right)-\Gamma_{k}\left(x_{i}, z\right) \Gamma_{l}\left(x_{i}, z\right)$, $1 \leq k \leq l \leq m$, is invertible. Thus, $b(z)$ and $a(z)$ are uniquely determined by (6). This holds for each $z \in \mathcal{D}$. By continuity of $b$ and $a$ hence for all $z \in \mathcal{Z}$.

Remark 2.4. Suppose that the STS (1) is used for daily estimation of the forward curve in terms of the state variable $z$. Then Proposition 2.3 tells us that, under the stated assumptions, any $\mathbb{Q}$-diffusion model $Z$ for $z$ is already determined by the choice of the functions $g$ and $\phi$. If $\mathcal{F}_{t}=\mathcal{F}_{t}^{W}$ is the Brownian filtration, then the diffusion coefficient of $Z$ is not affected by any Girsanov transformation. Consequently, statistical calibration is only possible for the drift of the model (or equivalently, for the market price of risk), since the observations of $z$ are made under the objective measure $\mathbb{P} \sim \mathbb{Q}$, where $d \mathbb{Q} / d \mathbb{P}$ is left unspecified by our consistency considerations. 
Define

$$
\begin{aligned}
B_{i}(z) & :=\sum_{k=1}^{m} b_{k}(z) \frac{\partial \phi_{i}(z)}{\partial z_{k}}+\frac{1}{2} \sum_{k, l=1}^{m} a_{k l}(z) \frac{\partial^{2} \phi_{i}(z)}{\partial z_{k} \partial z_{l}} \\
A_{i j}(z)=A_{j i}(z) & :=\frac{1}{2} \sum_{k, l=1}^{m} a_{k l}(z) \frac{\partial \phi_{i}(z)}{\partial z_{k}} \frac{\partial \phi_{j}(z)}{\partial z_{l}} .
\end{aligned}
$$

and notice that the right hand side of (6) can be rewritten

$$
\sum_{i=1}^{n}\left(g_{i}(x)-g_{i}(0)\right) \phi_{i}(z)=\sum_{i=1}^{n} G_{i}(x) B_{i}(z)-\sum_{i, j=1}^{n} G_{i}(x) G_{j}(x) A_{i j}(z) .
$$

Proposition 2.5. If the functions $G_{i}$ and $G_{i} G_{j}, 1 \leq i \leq j \leq n$, are linearly independent, then each $B_{i}$ and $A_{i j}$ can be expressed as a linear combination of $\phi_{1}, \ldots, \phi_{n}$.

Proof. Set $N=n+n(n+1) / 2$, the number of unknown functions $B_{i}$ and $A_{i j}$. There exists a sequence $0 \leq x_{1}<\cdots<x_{N}$ such that the $N \times N$-matrix with $k$-th row vector built by $G_{i}\left(x_{k}\right)$ and $G_{i}\left(x_{k}\right) G_{j}\left(x_{k}\right), 1 \leq i \leq j \leq n$, is invertible. In view of (9), the proposition follows.

Remark 2.6. In general, Proposition 2.5 fails without the linear independence assumption on $G$, see the example in Filipović (2001b).

Finally, we have the following regularity result.

Proposition 2.7. Suppose that the functions $\phi_{1}, \ldots, \phi_{n}$ are linearly independent. Then the $G_{i}$ s solve a system of Riccati equations. That is, for each $i \in\{1, \ldots, n\}$ there exists a polynomial of the form

$$
R_{i}(\lambda)=\gamma_{i}+\left\langle\beta_{i}, \lambda\right\rangle-\left\langle\alpha_{i} \lambda, \lambda\right\rangle, \quad \lambda \in \mathbb{R}^{n},
$$

where $\gamma_{i}=g_{i}(0), \beta_{i} \in \mathbb{R}^{n}$ and $\alpha_{i}$ is a symmetric $n \times n$-matrix, such that

$$
\frac{d G_{i}(x)}{d x}=R_{i}(G(x)) .
$$

Consequently, $g$ is analytic and uniquely determined by $\phi, b$ and $a$.

Proof. Choose mutually distinct points $z_{1}, \ldots, z_{n} \in \mathcal{Z}$ such that the $n \times n$-matrix $\left(\phi_{i}\left(z_{j}\right)\right)$ is regular. Now invert equation (9). This yields (10). Since the $R_{i}$ s are analytic functions, the second statement follows as well.

Corollary 2.8. Any STS model yields analytic forward curves.

Proof. We can always choose a collection $\mathcal{I}=\left\{\phi_{i_{1}}, \ldots, \phi_{i_{p}}\right\}, p \leq n$, which is linearly independent, such that any $\phi_{i}$ can be expressed as a linear combination of $\mathcal{I}$. But then (1) can be rewritten

$$
r(t, x)=\sum_{j=1}^{p} \tilde{g}_{j}(x) \phi_{i_{j}}\left(Z_{t}\right),
$$

for some continuous functions $\tilde{g}_{j}$. Now Proposition 2.7 yields the result. 


\section{The Maximal Degree Problem}

In this section we focus on the subclass of PTS models (2). To avoid trivialities we assume from now on that $n \geq 1$, since $n=0$ yields $r(t, x) \equiv$ const, see (6). For simplicity we consider only the time homogeneous case - the generalization of the subsequent results to time varying coefficients is straightforward. As before, $g, b$ and $\sigma$ are continuous.

First we shall prove a stronger version of Proposition 2.3. Recall Remark 2.4.

Proposition 3.1 (PTS). If $m=1$ then $b$ and $a$ are uniquely determined $b y$.

Proof. In view of Proposition 2.3 we have to show that there exists a dense subset $\mathcal{D}$ of $\mathcal{Z}$ such that $\Gamma(\cdot, z)$ and $\Lambda(\cdot, z)-\Gamma(\cdot, z)^{2}$ are linearly independent functions, for all $z \in \mathcal{D}$.

Hence, suppose there exists a closed set $\mathcal{N} \subset \mathcal{Z}$ with non-empty interior $\mathcal{N}^{0}$, such that

$$
c(z) \Gamma(\cdot, z)=\gamma(z)\left(\Lambda(\cdot, z)-\Gamma(\cdot, z)^{2}\right),
$$

for some $(c(z), \gamma(z)) \in \mathbb{R}^{2} \backslash\{(0,0)\}$, for all $z \in \mathcal{N}$. Fix $x \in \mathbb{R}_{+}$. Since $\Gamma(x, z)$ is analytic in $z$, without loss of generality $\Gamma(x, z) \neq 0$, for all $z \in \mathcal{N}^{0}$. Hence $\gamma$ does not vanish on $\mathcal{N}^{0}$, and we can set $\gamma(z) \equiv 1$. This implies

$$
c(z)=\frac{\sum_{i=2}^{n} i(i-1) G_{i}(x) z^{i-2}}{\sum_{i=1}^{n} i G_{i}(x) z^{i-1}}-\sum_{i=1}^{n} i G_{i}(x) z^{i-1}, \quad \forall z \in \mathcal{N}^{0}, \quad \forall x \in \mathbb{R}_{+},
$$

where $c$ is an analytic function in $\mathcal{N}^{0}$. Since the representation of the polynomial on the right hand side is unique, we have that $G_{i}(x) \equiv$ const $=G_{i}(0)=0$, for all $1 \leq i \leq n$. This implies $g_{i}=0$, for all $1 \leq i \leq n$, a contradiction.

We now shall show in two versions that, essentially, there exists no PTS model of degree $n>2$. The first version includes a result on the functional form of the coefficients $b$ and $a$.

We introduce the following notation: for $\mu \in\{1, \ldots, n\}$ and $k \in\{1, \ldots, m\}$ write $(\mu)_{k}$ for the multi-index with $\mu$ at the $k$-th position and zeros elsewhere. Let $\mathbf{i}_{1}, \mathbf{i}_{2}, \ldots, \mathbf{i}_{N}$ be a numbering of the set of multi-indices $I=\left\{\mathbf{i}=\left(i_{1}, \ldots, i_{m}\right)|| \mathbf{i} \mid \leq\right.$ $n\}$, where $N:=|I|=\sum_{|\mathbf{i}|=0}^{n} 1$.

Theorem 3.2 (Maximal Degree Problem I). Suppose that $G_{\mathbf{i}_{\mu}}$ and $G_{\mathbf{i}_{\mu}} G_{\mathbf{i}_{\nu}}$ are linearly independent functions, $1 \leq \mu \leq \nu \leq N$, and that $\sigma \not \equiv 0$.

Then necessarily $n \in\{1,2\}$. Moreover, $b(z)$ and $a(z)$ are polynomials in $z$ with $\operatorname{deg} b(z) \leq 1$ in any case (QTS and ATS), and $\operatorname{deg} a(z)=0$ if $n=2$ (QTS) and $\operatorname{deg} a(z) \leq 1$ if $n=1$ (ATS).

Proof. Proposition 2.5 yields that each of the expressions $B_{\mathbf{i}}(z)$ and $A_{\mathbf{i j}}(z)$ is a polynomial in $z$ of order less than or equal $n$, see (7) and (8). In particular, we have

$$
\begin{aligned}
B_{(1)_{k}}(z) & =b_{k}(z), \\
2 A_{(1)_{k}(1)_{l}}(z) & =a_{k l}(z), \quad k, l \in\{1, \ldots, m\},
\end{aligned}
$$

hence $b(z)$ and $a(z)$ are polynomials in $z$ with $\operatorname{deg} b(z), \operatorname{deg} a(z) \leq n$. An easy calculation shows that

$$
2 A_{(n)_{k}(n)_{k}}(z)=a_{k k}(z) n^{2} z_{k}^{2 n-2}, \quad k \in\{1, \ldots, m\} .
$$


We may assume that $a_{k k} \not \equiv 0$, since $\sigma \not \equiv 0$. But then the right hand side of (12) cannot be a polynomial in $z$ of order less than or equal $n$ unless $n \leq 2$. This proves the first part of the theorem.

If $n=1$ there is nothing more to prove. Now let $n=2$. Notice that by definition

$$
\operatorname{deg}_{\mu} a_{k l}(z) \leq\left(\operatorname{deg}_{\mu} a_{k k}(z)+\operatorname{deg}_{\mu} a_{l l}(z)\right) / 2,
$$

where $\operatorname{deg}_{\mu}$ denotes the degree of dependence on the single component $z_{\mu}$. Equation (12) yields $\operatorname{deg}_{k} a_{k k}(z)=0$. Hence $\operatorname{deg}_{l} a_{k l}(z) \leq 1$. Consider

$$
2 A_{(1)_{k}+(1)_{l},(1)_{k}+(1)_{l}}(z)=a_{k k}(z) z_{l}^{2}+2 a_{k l}(z) z_{k} z_{l}+a_{l l}(z) z_{k}^{2}, \quad k, l \in\{1, \ldots, m\} .
$$

From the preceding arguments it is now clear that also $\operatorname{deg}_{l} a_{k k}(z)=0$, and hence $\operatorname{deg} a(z)=0$. We finally have

$$
B_{(1)_{k}+(1)_{l}}(z)=b_{k}(z) z_{l}+b_{l}(z) z_{k}+a_{k l}(z), \quad k, l \in\{1, \ldots, m\},
$$

from which we conclude that $\operatorname{deg} b(z) \leq 1$.

Remark 3.3. The preceding result on QTS models has already been stated in Leippold and Wu (2002). There, however, the precise conditions are left unspecified.

We can relax the hypothesis on $G$ in Theorem 3.2 if from now on we make the following standing assumptions: $\mathcal{Z} \subset \mathbb{R}^{m}$ is a cone, and $b$ and $\sigma$ satisfy a linear growth condition

$$
\|b(z)\|+\|\sigma(z)\| \leq C(1+\|z\|), \quad \forall z \in \mathcal{Z},
$$

for some constant $C \in \mathbb{R}_{+}$.

Theorem 3.4 (Maximal Degree Problem II). Suppose that

$$
\langle a(z) v, v\rangle \geq k(z)\|v\|^{2}, \quad \forall v \in \mathbb{R}^{m},
$$

for some function $k: \mathcal{Z} \rightarrow \mathbb{R}_{+}$with

$$
\liminf _{z \in \mathcal{Z},\|z\| \rightarrow \infty} k(z)>0 .
$$

Then necessarily $n \in\{1,2\}$.

Conditions (14) and (15) say that $a(z)$ becomes uniformly elliptic for $\|z\|$ large enough.

Proof. We shall make use of the basic inequality

$$
\left|z^{\mathbf{i}}\right| \leq\|z\|^{|\mathbf{i}|}, \quad \forall z \in \mathbb{R}^{m} .
$$

This is immediate, since

$$
\frac{\left|z^{\mathbf{i}}\right|}{\|z\|^{|\mathbf{i}|}}=\left(\frac{z_{1}}{\|z\|}\right)^{i_{1}} \cdots\left(\frac{z_{m}}{\|z\|}\right)^{i_{m}} \leq 1, \quad \forall z \in \mathbb{R}^{m} \backslash\{0\} .
$$

Suppose now that $n>2$. We have from (4)

$$
\Gamma_{k}(x, z)=\sum_{|\mathbf{i}|=n} G_{\mathbf{i}}(x) i_{k} z^{\mathbf{i}-(1)_{k}}+\cdots=: P_{k}(x, z)+\cdots,
$$

where $P_{k}(x, z)$ is a homogeneous polynomial in $z$ of order $n-1$, and $\cdots$ stands for lower order terms in $z$. By assumptions there exist $x \in \mathbb{R}_{+}$and $k \in\{1, \ldots, m\}$ such that $P_{k}(x, \cdot) \neq 0$. Choose $z^{*} \in \mathcal{Z} \backslash\{0\}$ with $P_{k}\left(x, z^{*}\right) \neq 0$ and set $z_{\alpha}:=\alpha z^{*}$, for $\alpha>0$. Then we have $z_{\alpha} \in \mathcal{Z}$ and

$$
\Gamma_{k}\left(x, z_{\alpha}\right)=\alpha^{n-1} P_{k}\left(x, z^{*}\right)+\cdots,
$$


where $\cdots$ denotes lower order terms in $\alpha$. Consequently,

$$
\lim _{\alpha \rightarrow \infty} \frac{\Gamma_{k}\left(x, z_{\alpha}\right)}{\left\|z_{\alpha}\right\|^{n-1}}=\frac{P_{k}\left(x, z^{*}\right)}{\left\|z^{*}\right\|^{n-1}} \neq 0 .
$$

Combining (14) and (15) with (17) we conclude that

$$
L:=\liminf _{\alpha \rightarrow \infty} \frac{1}{\left\|z_{\alpha}\right\|^{2 n-2}}\left\langle a\left(z_{\alpha}\right) \Gamma\left(x, z_{\alpha}\right), \Gamma\left(x, z_{\alpha}\right)\right\rangle \geq \liminf _{\alpha \rightarrow \infty} k\left(z_{\alpha}\right) \frac{\left\|\Gamma\left(x, z_{\alpha}\right)\right\|^{2}}{\left\|z_{\alpha}\right\|^{2 n-2}}>0 .
$$

On the other hand, by (6),

$$
L \leq \sum_{|\mathbf{i}|=0}^{n}\left|g_{\mathbf{i}}(x)-g_{\mathbf{i}}(0)\right| \frac{\left|z_{\alpha}^{\mathbf{i}}\right|}{\left\|z_{\alpha}\right\|^{2 n-2}}+\frac{\left\|b\left(z_{\alpha}\right)\right\|}{\left\|z_{\alpha}\right\|} \frac{\left\|\Gamma\left(x, z_{\alpha}\right)\right\|}{\left\|z_{\alpha}\right\|^{2 n-3}}+\frac{1}{2} \frac{\left\|a\left(z_{\alpha}\right)\right\|}{\left\|z_{\alpha}\right\|^{2}} \frac{\left\|\Lambda\left(x, z_{\alpha}\right)\right\|}{\left\|z_{\alpha}\right\|^{2 n-4}},
$$

for all $\alpha>0$. In view of (4), (5), (13) and (16), the right hand side converges to zero for $\alpha \rightarrow \infty$. This contradicts (18), hence $n \leq 2$.

Remark 3.5. Notice that the preceding setup (except of (13)) is always satisfied for Gaussian models.

Finally, we consider a seemingly paradox example. It both clarifies the above assumptions and exposes "pseudo" PTS models of higher order. Let $\left(R_{t}\right)$ be the short rate process

$$
d R_{t}=\sqrt{R_{t}} d W_{t}, \quad R_{0} \geq 0
$$

where $W$ is a real-valued Brownian motion. This is a simplified version of the CoxIngersoll-Ross short rate model (Cox et al. (1985)), and it is well known that $R_{t}$ is nonnegative and yields an ATS of the form

$$
r(t, x)=h(x) R_{t}
$$

(for $h$ see e.g. Section 7.4.1 in Filipović (2001a)). Now write $Z_{t}=\left(R_{t}\right)^{1 / 3}$. Then

$$
r(t, x)=h(x)\left(Z_{t}\right)^{3}
$$

is a consistent PTS model of degree 3. Does this contradict the preceding theorems?

The answer is of course no, and the reason is that the assumptions for both Theorems 3.2 and 3.4 are not satisfied. First, notice that the full form of (19) reads

$$
r(t, x)=g_{0}(x)+g_{1}(x) Z_{t}+g_{2}(x)\left(Z_{t}\right)^{2}+g_{3}(x)\left(Z_{t}\right)^{3},
$$

with $g_{0}=g_{1}=g_{2}=0$ and $g_{3}=h$. But this means that the functions $G_{0}, \ldots, G_{3}$, see (3), are not linearly independent. Whence Theorem 3.2 does not apply. Second, we have

$$
d Z_{t}=-\frac{1}{9\left(Z_{t}\right)^{2}} d t+\frac{1}{3 \sqrt{Z_{t}}} d W_{t}
$$

Hence the diffusion coefficient is $a(z)=1 /(9 z)$, which does not satisfy (14) and (15).

\section{Conclusion}

We have explored the interplay between the diffusion coefficients and the functions determining the forward curve for an arbitrage-free STS model. For QTS models the diffusion is, under the appropriate conditions, necessarily an Ornstein-Uhlenbeck type process. We recaptured also the ATS models. Moreover, we have solved the maximal degree problem: under reasonable conditions, the only consistent PTS models are the ATS and QTS models. 
Acknowledgement. I am grateful to W. Runggaldier for having brought the maximal degree problem to my attention.

Financial support from Credit Suisse is gratefully acknowledged.

\section{REFERENCES}

[1] P. Boyle, and W. Tian, Quadratic interest rate models as approximations to effective rate models, Working paper, University of Waterloo, 1999.

[2] J. Cox, J. Ingersoll, and S. Ross, A theory of the term structure of interest rates, Econometrica 53 (1985), 385-408.

[3] D. Duffie, D. Filipović, and W. Schachermayer, Affine processes and applications in finance, Working Paper, 2001.

[4] D. Duffie and R. Kan, A yield-factor model of interest rates, Math. Finance 6 (1996), 379-406.

[5] D. Duffie, J. Pan, and K. Singleton, Transform analysis and asset pricing for affine jumpdiffusions, Econometrica 68 (2000), no. 6, 1343-1376.

[6] D. Filipović, Consistency problems for Heath-Jarrow-Morton interest rate models, SpringerVerlag, Berlin, 2001a.

[7] D. Filipović, A general characterization of one factor affine term structure models, Finance Stoch. 5 (2001b), no. 3, 389-412.

[8] A. Gombani and W. J. Runggaldier, A filtering approach to pricing in multifactor term structure models, Working paper, University of Padova, 2000.

[9] D. Heath, R. Jarrow, and A. Morton, Bond pricing and the term structure of interest rates: A new methodology for contingent claims valuation, Econometrica 60 (1992), 77-105.

[10] M. Leippold and L. Wu, Asset pricing under the quadratic class, forthcoming in Journal of Financial and Quantitative Analysis (2002).

Department of Operations Research and Financial Engineering, Princeton UniverSity, Princeton, NJ 08544, USA

E-mail address: dfilipov@princeton.edu 